\section{Monitoramento e avaliação na atenção básica: novos horizontes}

\author{
Monitoring and evaluation in primary \\ health care: new perspectives
}

Eronildo Felisberto 1

1 Coordenação de Acompanhamento e Avaliação.

Departamento de Atenção Básica. Secretaria de Atenção à Saúde. Ministério da Saúde. Esplanada dos Ministérios, Bloco G, 6.andar, sala 635. Brasília, DF, Brasil. CEP: 70.058-900
Estão claras e não são de hoje as proposições e posições assumidas por gestores e pesquisadores que têm refletido sobre a necessidade de se investir na institucionalização da avaliação.1-11 Entendendo Institucionalização enquanto incorporação da avaliação à rotina dos serviços, estamos assumindo a necessidade do fortalecimento e/ou desenvolvimento de capacidade técnica, nos diversos níveis do sistema de saúde, para adotar as ações de monitoramento e avaliação como subsidiárias ou intrínsecas ao planejamento e à gestão, como instrumento de suporte à formulação de políticas, ao processo decisório e de formação dos sujeitos envolvidos (gestores, usuários do sistema de saúde e profissionais dos serviços e das instituições de ensino e pesquisa).

Investir na institucionalização da avaliação deve ser entendido aqui como contribuição decisiva com o objetivo de qualificar a atenção básica, promovendo-se a construção de processos estruturados e sistemáticos, coerentes com os princípios do Sistema Único de Saúde (universalidade, equidade, integralidade, participação social, resolutividade, acessibilidade) e, abrangentes em suas várias dimensões - da gestão, do cuidado e do impacto sobre o perfil epidemiológico.

A avaliação da situação de saúde da população, do trabalho em saúde e dos resultados das ações, decorre em grande parte das atividades de monitoramento realizadas a partir das informações produzidas no cotidiano da atenção. Essas, embora sejam insuficientes para apreender todas as mudanças desejáveis, são essenciais para orientação dos processos de implantação, consolidação e reformulação das práticas de saúde.

Por outro lado, a avaliação de políticas e programas de saúde deve contemplar ampla participação e o uso de múltiplos focos e métodos, permitindo que a visão de diferentes grupos seja considerada no objeto de estudo. Daí a necessidade de estudos e pesquisas que dêem conta das dimensões não apreendidas pelos diversos sistemas de monitoramento.

O desafio à consolidação do SUS estabelecido pela Norma Operacional da Assistência à Saúde $(\mathrm{NOAS} / 2001)^{12}$ com vistas à construção de redes assistenciais em atenção básica eficazes e resolutivas, integralmente coesas com os níveis de atenção mais complexos, prevê o desenvolvimento de mecanismos que visem a garantia de qualidade. Isso posto, constitui-se de fundamental importância priorizar a qualificação das equipes técnicas para a apreensão de práticas e capacidades específicas mas, também, para a aquisição de competências avaliativas.

Para Hartz: (2002: 419). ${ }^{9}$

"... Institucionalizar a avaliação tem o sentido de integrá-la em um sistema organizacional no qual esta seja capaz de influenciar o seu comportamento, ou seja, um modelo orientado para a ação ligando necessariamente as atividades analíticas às de gestão ..."

Este Informe foi publicado anteriormente na Revista Brasileira de Saúde da Família 2003; 5 (7): 24-29.

[edição especial]. (ISSN 1518-2355). 
O Programa Saúde da Família (PSF) vem se constituindo, ao longo do tempo, como indutor desse processo de institucionalização da avaliação na atenção básica. ${ }^{6}$ A criação, na estrutura organizacional do Departamento de Atenção Básica da Secretaria de Atenção à Saúde do Ministério da Saúde (DAB/SAS/MS) de uma Coordenação de Acompanhamento e Avaliação (CAA) marca essa característica, principalmente quando se atribui a essa a responsabilidade da condução gerencial de iniciativas processuais de grande envergadura como o "Pacto de Indicadores da Atenção Básica", estabelecido mediante negociação entre as três esferas de gestão, que tem se constituído num esforço coletivo de incorporação da avaliação à prática da gestão e de articulação com os processos de programação; a Investigação Avaliativa representada pela realização de estudos e pesquisas de abrangência nacional e o Sistema de Informação da Atenção Básica (SIAB), principal instrumento de monitoramento da atenção básica com características muito peculiares de apoio à gestão, ao fazer uso dos conceitos de territorialização, adscrição de clientela e potencialidade de uso por profissionais integrantes das equipes de saúde para discussão com a comunidade usuária e programação local.4,10

Entretanto, para o sistema nacional de saúde, em especial no campo da atenção básica, ainda buscase clarear as diretrizes de uma política de avaliação, que embora em franco processo de implantação, estabelece como desafio a superação dos obstáculos operacionais e funcionais para sua execução. $\mathrm{Na}$ atual gestão do Ministério da Saúde, coube ao Departamento de Atenção Básica da Secretaria de Atenção à Saúde, por intermédio da Coordenação de Acompanhamento e Avaliação, propor estratégias e desencadear ações para o enfrentamento a esse desafio.

Uma primeira iniciativa tomada por essa Coordenação foi a definição de sua missão institucional e a identificação clara de seus objetivos estratégicos que, a despeito de possíveis limitações, tem sua formulação resultante de um processo participativo em seu âmbito, permitindo, dessa forma, traçar as operações a serem desenvolvidas com o fim de atingi-los. (Quadro 1)

\section{Quadro 1}

Missão e objetivos estratégicos da Coordenação de Acompanhamento e Avaliação.

\section{Missão}

Monitorar e avaliar a atenção básica instrumentalizando a gestão e fomentar/consolidar a cultura avaliativa nas três instâncias de gestão do Sistema Único de Saúde (SUS).

\section{Objetivos estratégicos}

Identificar aspectos relevantes da atenção básica para serem monitorados e avaliados, com destaque para o Programa Saúde da Família (PSF);

Desenvolver estratégias de disseminação das informações relevantes, precisas e de forma ágil sobre Atenção Básica $(A B)$ no País;

Construir capacidade técnica e política nas três instâncias gestoras do SUS que possibilite a análise dos dados para a tomada de decisão;

Induzir processos de articulação intra e intersetorial visando a institucionalização do Monitoramento e da Avaliação da Atenção Básica;

Fomentar e dinamizar estratégias de pactuação de metas e indicadores da $A B$ nas três instâncias de gestão do Sistema Único de Saúde;

Garantir o acesso aos bancos de dados sob gerência da coordenação para monitorar e avaliar a Atenção Básica;

Identificar a necessidade de fomentar e articular estudos avaliativos para a Atenção Básica;

Gerenciar o Sistema de Informação da Atenção Básica. 
O passo seguinte constituiu-se em viabilizar espaços para discussão das bases e diretrizes para a promoção de uma cultura avaliativa - envolvendo os diferentes atores por intermédio de referências conceituais, metodológicas e operacionais - que contribua para a implementação de mecanismos e instâncias de avaliação da atenção básica nos níveis local e regional e que estimule uma reflexão mais sistematizada sobre a institucionalização da avaliação.

Dessa forma, alguns movimentos vêm ocorrendo com o objetivo de se pactuar os aspectos importantes da atenção básica a serem monitorados e avaliados, a partir de um processo de mobilização de técnicos, gestores e pesquisadores, buscando-se, também, manter aceso o debate sobre a necessidade do estabelecimento de padrões de qualidade para a atenção básica, partindo-se da estratégia saúde da família.

Por outro lado, superar o desafio de articular os diversos projetos e ações propostos e/ou em execução como "ferramentas" para o monitoramento e avaliação da atenção básica constituem hoje o estado da arte da construção de uma política de avaliação para a atenção básica no Brasil.

É nesse sentido que, a partir do debate mais ampliado no âmbito do Ministério da Saúde e da Comissão Intergestora Tripartite, se procurou definir as estratégias de ação necessárias a dar organicidade ao conjunto das diversas iniciativas e processos de trabalho em curso.

A reformulação do SIAB com o objetivo de transformá-lo num sistema que seja abrangente de toda a atenção básica, superando os limites do Programa de Agentes Comunitários de Saúde (PACS) e do PSF; a dinamização do Pacto de Indicadores da Atenção Básica como instrumento de mobilização, de negociação política mas, efetivamente, como elemento norteador da formulação das políticas setoriais e da programação das ações; a divulgação de forma ampla dos estudos e pesquisas realizados sobre a atenção básica, proporcionando o acesso de gestores, profissionais dos serviços de saúde e docentes, pesquisadores e estudantes das instituições de ensino e pesquisa; a disponibilização pública do banco de dados do SIAB; a exploração dos sistemas de informação e outras fontes de dados produzindose indicadores e analisando-os com vistas a publicações institucionais; a construção de instrumentos que permitam o uso mais sistemático da informação pelas equipes de saúde e gestores do sistema; a divulgação de experiências exitosas implementadas por estados e municípios; a realização de novos estudos e pesquisas como necessidade à complementação do monitoramento e a identificação de fontes de financiamento e definição de orçamento para as ações de avaliação, são algumas das estratégias que vêm sendo efetivadas pelo Ministério da Saúde.

Paralelamente, se fez necessário a renegociação do acordo de empréstimo assinado entre o governo brasileiro e o Banco Internacional para Reconstrução e Desenvolvimento (BIRD - Banco Mundial) que originou o Projeto de Expansão e Consolidação do Programa Saúde da Família (PROESF), no que concerne ao Componente III - Monitoramento e Avaliação, tanto no que diz respeito aos seus subcomponentes e/ou ações programadas, quanto no que se refere ao volume de recursos destinados à sua execução.

A inclusão da proposta de Fortalecimento da Capacidade Técnica das Secretarias Estaduais de Saúde em Monitoramento e Avaliação e o aumento dos recursos financeiros de U\$ 7 milhões para U\$ 25 milhões previstos para a primeira fase do PROESF no Componente III, se caracterizam como elementos direcionados a dar corpo à superação daquele desafio identificado para a definição da política, constituindo fatores estruturadores da sustentabilidade para a construção da capacidade avaliativa em saúde para o SUS.

Da mesma forma, o fomento ao debate interno sobre a necessidade da integração das práticas de monitoramento e avaliação e sua articulação com o planejamento e a formulação das políticas setoriais, com vistas a uma repercussão uníssona nas programações e pactuações, tem resultado em ocupação de importante espaço da avaliação no planejamento macroestratégico das ações de saúde.

A conjunção de decisão político-institucional, recursos financeiros, mecanismos técnicos e estratégias organizacionais de qualificação dos recursos humanos para avaliação constitui o eixo para a construção da capacidade institucional, o que, sem sombra de dúvidas, vem superar a prática de se estabelecer processos avaliativos unicamente atrelados à intervenção. ${ }^{11}$ A complexidade do SUS representada pelo processo de descentralização da gestão, prevê o envolvimento de atores e contextos locais diversificados, gerando a redefinição de papéis, responsabilidades e necessidades de investimento, o que se acredita estar sendo contemplado a partir das proposições anteriormente referidas. 1

A proposição, portanto, aqui ressaltada e, que no nosso entendimento passa a se constituir no eixo estruturante do delineamento da política de avaliação para a atenção básica no país, toma corpo a partir da construção pelas Secretarias Estaduais de Saúde (SES) dos Planos Estaduais para Fortalecimento das Ações de Monitoramento e Avaliação da Atenção Básica. 
Esses planos, no momento em fase de elaboração, têm como referência os pressupostos conceituais e diretrizes propostos pelo Ministério da Saúde e pactuados na Comissão Intergestora Tripartite que também definiu os indicadores para a elaboração dos mesmos, assim como os critérios técnicos para o financiamento das ações propostas. 13

Tendo como forma de financiamento a transferência direta de recursos financeiros do Fundo Nacional de Saúde para o Fundo Estadual de Saúde, esse se dará mediante a avaliação do cumprimento de metas previamente estabelecidas e aprovadas pelas instâncias colegiadas de gestão e de controle social.

A necessidade de conferir maior organicidade institucional aos processos de avaliação a serem desenvolvidos prevê que as propostas apresentadas estejam pautadas em algumas linhas norteadoras que obedecem aos princípios do SUS e as diretrizes do PSF enquanto estratégia prioritária para a organização das práticas e serviços de atenção básica. 13

São U\$ 17 milhões destinados as SES para a primeira fase do projeto e alguns indicadores estão sendo negociados com vistas a garantir recursos financeiros durante todo o período de vigência do
PROESF - cerca de seis anos, tempo suficiente, ao nosso ver, para consolidação desta ação com o desenvolvimento de estratégias de sustentabilidade e construção de estruturas permanentes de avaliação no âmbito dos estados e a conseqüente capilarização da idéia para as gestões municipais, guardando coerência com os estágios de desenvolvimento, amadurecimento e capacidades técnicas evidenciadas pelo processo de descentralização da gestão do SUS.

A construção e/ou fortalecimento da capacidade técnica em monitoramento e avaliação, com vistas a sua inserção firme e decisiva nas práticas de saúde, seja do cuidado ou da gestão, remete-nos a longos caminhos a serem percorridos. Entretanto, o que aqui está se apresentando e pondo-se em discussão tratase de uma estratégia clara, objetiva, como forma de se trazer à prática do cotidiano, ao campo do real e com direcionalidade às necessidades de saúde da população, as reflexões e proposições teóricas da disciplina avaliação, tomando como referência desde as clássicas abordagens concebidas por Donabedian 14 até as proposições de sua aplicabilidade por alguns autores que vêm contribuindo de forma importante para essa construção.7,15-18

\section{Referências}

1. Hartz ZMA, organizadora. Avaliação em saúde: dos modelos conceituais à prática na análise da implantação de programas. Rio de Janeiro: FIOCRUZ; 1997.

2. Schraiber LB, Peduzzi M, Sala A, Nemes MIB, Castanhera ERL, Kon R. Planejamento, gestão e avaliação em saúde: identificando problemas. Cienc Saúde Coletiva 1999; 4: 221-2.

3. Vieira-da-Silva LM. The field of evaluation and the "sur mesure" strategy. Cad Saúde Pública 1999; 15: 247-8.

4. Hartz ZMA. Pesquisa em avaliação da atenção básica: a necessária complementação do monitoramento. Div Saúde Debate 2000; 21: 29-35.

5. Medina MG, Aquino R, Carvalho ALB. Avaliação da atenção básica: construindo novas ferramentas para o SUS. Div Saúde Debate 2000; 21: 15-28.

6. Ministério da Saúde. Saúde da família no Brasil - linhas estratégicas para o quadriênio 1999/2002. Brasília (DF) Secretaria de Políticas de Saúde, Departamento de Atenção Básica; 2000

7. Scriven M. Evaluation: future tense. Am J Eval 2001; 22: 301-7.

8. Bodstein R. Atenção básica na agenda da saúde. Cienc Saúde Coletiva 2002; 7: 401-12.
9. Hartz ZMA. Institucionalizar e qualificar a avaliação: outros desafios para a atenção básica. Cienc Saúde Coletiva 2002; 7: 419-21.

10. Souza HM. O PSF como indutor da institucionalização da avaliação na atenção básica. Rev Bras Saúde Fam 202; 6: $10-5$.

11. Stevenson JF, Florin P, Mills DS, Andrade M. Building evaluation capacity in human service organizations: a case study. Eval Progr Plan 2002; 25: 233-43.

12. Ministério da Saúde. Norma Operacional da Assistência à Saúde. Sistema Único de Saúde (NOAS/SUS). Portaria $\mathrm{n}^{\circ} 95$ de 26 de janeiro de 2001. Diário Oficial da União [DOU] 2001 Jan 29; Sec 1, 139 (20E): 23-31.

13. Ministério da Saúde. Planos estaduais para fortalecimento das Ações de Monitoramento e Avaliação da Atenção Básica - Diretrizes e Orientações. Brasília (DF): Secretaria de Atenção à Saúde, Departamento de Atenção Básica, Coordenação de Acompanhamento e Avaliação; 2004. [versão preliminar].

14. Donabedian A. The quality of care-how an it be assessed? JAMA 1988; 260: 1743-8.

15. Contandriopoulos AP, Champagne F, Denis JL, Pineault R. A Avaliação na área da saúde: conceitos e métodos. In: 
Harzt ZMA, organizadora. Avaliação em saúde: dos modelos conceituais à prática na análise da implantação de programas. Rio de Janeiro: FIOCRUZ; 1997. p. 2947.

16. Vieira-da-Silva LM, Formigli VLA. Avaliação em saúde limites e perspectivas. Cad Saúde Pública 1994; 10: 8091.

17. Hartz ZMA. Institucionalizing the evaluation of health programs and policies in France: cuisine internationale over fast food and sur mesure over ready-made. Cad Saúde Pública 1999; 15: 229-59.

18. Patton MQ. Evaluation, knowledge management, best pratices, and high quality: lessons learned. Am J Evaluat 2001; 22: 329-36.

Recebido em 8 de março de 2004

Versão final apresentada em 9 de junho de 2004

Aprovado em 22 de julho de 2004 